\title{
Deuterium Retention in Plasma Sprayed Tungsten Exposed to Low Energy Deuterium Plasma
}

\author{
Aleksandr RUSINOV, Mizuki SAKAMOTO ${ }^{1)}$, Hideki ZUSHI ${ }^{2)}$, Ryohei OHYAMA, \\ Koichiro HONDA, Tetsuo TANABE and Naoaki YOSHIDA ${ }^{2)}$ \\ Interdisciplinary Graduate School of Engineering Sciences, Kyushu University, Kasuga 816-8580, Japan \\ 1) Plasma Research Center, University of Tsukuba, Tsukuba 305-8577, Japan \\ 2) Research Institute for Applied Mechanics, Kyushu University, Kasuga 816-8580, Japan
}

(Received 5 December 2011 / Accepted 19 November 2012)

\begin{abstract}
Deuterium retention and trapping in tungsten plasma sprayed layers after low energy plasma irradiation was investigated by means of thermal desorption technique. Plasma irradiation at low temperatures $(<650 \mathrm{~K})$ showed the retention and trapping similar to polycrystalline tungsten material. On the other hand, irradiation at high temperatures $(>650 \mathrm{~K}$ ) showed significant increase of the retention in the plasma sprayed layers contrary to the polycrystalline material. Thermal desorption spectra showed extremely high temperatures of the peak positions which are non-typical for the tungsten materials. The possible reasons for such high deuterium retention in the plasma sprayed layers after irradiation at high temperatures and the possible trapping mechanisms are discussed. (c) 2013 The Japan Society of Plasma Science and Nuclear Fusion Research
\end{abstract}

Keywords: tungsten, TDS, deuterium retention

DOI: $10.1585 /$ pfr.8.1405004

\section{Introduction}

Tungsten material is one of candidates for the divertor plates in the future thermonuclear experimental reactor ITER. Because of difficult machining and welding of bulk tungsten, plasma sprayed (PS) tungsten (W) layers, which have been used in ASDEX and other tokamaks, are also considered as plasma facing materials. It has several advantages such as low cost and possibility of coating of large areas even of non-planar shapes [1]. Although a lot of studies on hydrogen isotopes retention have been done with polycrystalline tungsten, there are few studies for the PS W layers. Literature data show large scattering for plasma exposed PS W, retention mechanisms haven't been investigated in details. Because divertor materials will be exposed to high flux plasma and heated to high temperatures, investigations under these conditions are of great interest. PS W has been used in the divertor plates and inner guard limiters of the QUEST spherical tokamak in Kyushu University [2]. It is necessary to study the retention characteristics of the material in the laboratory conditions in order to predict its behavior in tokamaks. In this study, the deuterium retention in PS W layers, exposed to low energy D plasma, was investigated at different irradiation temperatures $\left(T_{\text {irr }}\right)$ and fluences by means of thermal desorption spectroscopy (TDS).

\section{Experimental}

Samples used in the study were as received VPS (vacuum PS) and APS (atmospheric PS) W layers with the thickness of $150 \mu \mathrm{m}$ deposited on $1 \mathrm{~mm}$ F82H stainless steel substrate, prepared by TOCALO corp. SEM images of the surface relief of as received APS W and VPS W are shown in Fig. 1. It is seen, that the materials consist of W droplets with lot of pores and cracks between them. The porosity of APS W and VPS W layers assessed from the surface images of polished samples was about $5 \%$ and $3 \%$, respectively. Energy dispersive analysis (EDS) measurements of as received samples showed enhanced oxygen concentration on the surface at the level of about $50 \%$ and 20\% for APS W and VPS W, respectively. Experiments have been carried out with as received as well as with polished samples. The polishing process included mechanical polishing with subsequent chemical polishing and outgassing in TDS stand by usual TDS heating routine. Polycrystalline W (PCW) sample with the purity of $99.99 \%$ and the dimensions of $\varnothing 10 \times 1 \mathrm{~mm}$, produced by A.L.M.T. corp. was used in the experiments for comparison. It had been polished mechanically to a mirror like surface and annealed at $900 \mathrm{C}$ during 1 hour in vacuum prior to the first irradiation.

The samples were exposed to low energy D plasma in the Advanced PWI Simulation Experimental Device and Analysis System (APSEDAS). The scheme of the installation is shown in Fig. 2. The plasma in APSEDAS is produced by $13.56 \mathrm{MHz}$ RF field in a steady state magnetic field. The samples were mounted on a copper water cooled stage and exposed to the various fluences up to $10^{26} \mathrm{D} / \mathrm{m}^{2}$ under the similar fluxes of about $3 \times 10^{21} \mathrm{D} / \mathrm{m}^{2} \mathrm{sec}$. The stage was grounded and the energy of ions coming from 

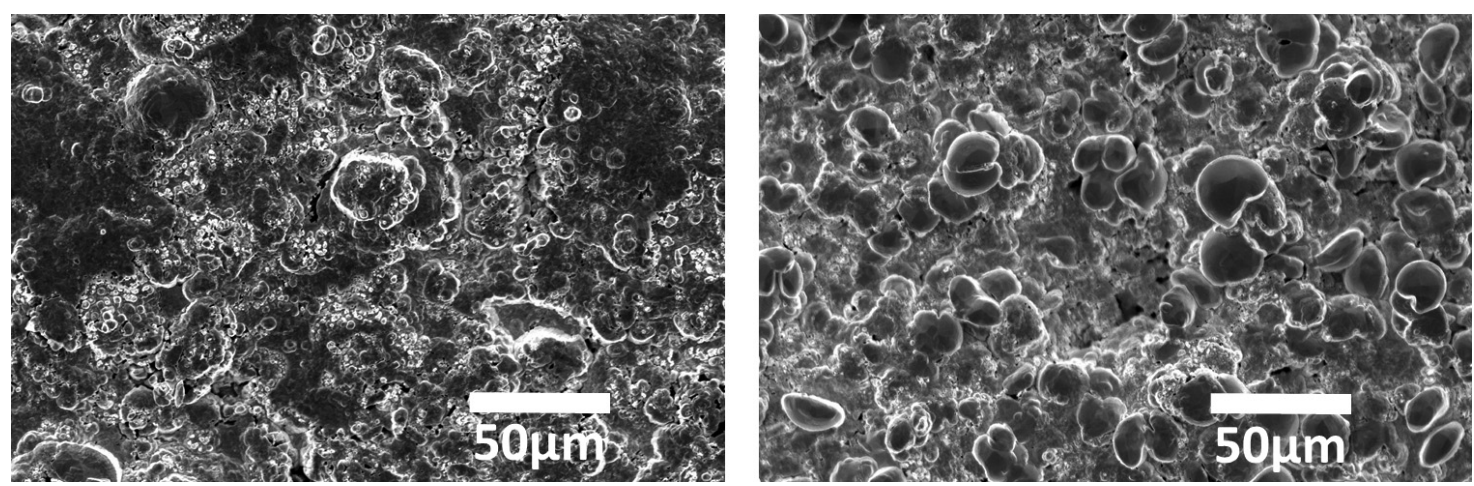

Fig. 1 SEM pictures of the surface relief of as received APS W (left) and VPS W (right) samples.



Fig. 2 APSEDAS PWI simulator scheme.

the plasma to the surface of the sample was estimated to be about $30 \mathrm{eV}$ according to the Langmuir probe measurements. During the irradiation the samples were heated by intense plasma particle fluxes and the surface temperature was measured by an infrared camera. The temperature was varied in the experiments using additional substrates in order to change thermal contact of a sample with the stage.

Thermal desorption was performed in a separate stand. The sample was installed on a tantalum bed with attached thermocouple. Then the bed was moved and evacuated inside a quartz tube and heated by an infrared oven from $300 \mathrm{~K}$ up to $1473 \mathrm{~K}$ with the rate of $1 \mathrm{~K} / \mathrm{sec}$. The desorbed species were detected by a quadrupole mass spectrometer. The calibration was performed in each experiment using He leak bottle taking into account once measured ratio of ionization cross-sections $\sigma_{\mathrm{He}+} / \sigma_{\mathrm{D} 2+}$. The amount of desorbed deuterium was calculated as an inte- gral of $\mathrm{D}_{2}$ and $\mathrm{HD}$ desorption signals over the time. HD calibration factor was assumed to be the same as that of $\mathrm{D}_{2}$.

Lower thermal conductivity of PS W layers might cause a certain concern about the temperature distribution inside the samples during the plasma irradiation and TDS heating. The temperature of deeper regions might differ from that on the surface. Simple calculations of the maximum temperature difference at the given experimental conditions showed value an order of 2 - 3 degree for the thermal conductivity of 100 times smaller than that of polycrystalline W. Therefore, errors in the temperature measurements during the irradiations and in TDS peak positions seem to be negligible.

\section{Results and Discussion}

The thermal desorption spectra (TDS) of deuterium for PCW, VPS W and APS W after irradiation at different $T_{\text {irr }}$ up to the fluence of $2 \times 10^{25} \mathrm{D} / \mathrm{m}^{2}$ are shown in Fig. 3. At low $T_{\text {irr }}(500 \mathrm{~K})$ TDS spectra for all three materials have the main peak near $800 \mathrm{~K}$. TDS spectra from APS and VPS W have noticeable high temperature part (900 $1300 \mathrm{~K}$ ) though its contribution to the total retention is relatively small. TDS spectra from PCW have negligible desorption in this TDS temperature region. The contribution of the low temperature part in TDS spectra (below $900 \mathrm{~K}$ ) decreases for all materials with the increase of $T_{\text {irr }}$. On the other hand, the contribution of high temperature part increases for PS W layers and isn't changed for PCW. The structure of the high temperature part is complicate in the case of PS W layers and consists of many desorption peaks with different amplitudes. The relative amplitudes of the peaks in this region are different for APS and VPS W samples though the peak positions are similar. Figure 4 shows the behavior of TDS spectra of deuterium from APS W for $T_{\text {irr }}=750 \mathrm{~K}$ with the fluence changing. It is seen that all peak amplitudes grow with the fluence whereas the relative amplitudes aren't changed much.

Figure 5 shows the dependence of the deuterium retention on $T_{\text {irr }}$ in PCW, APS and VPS W layers, measured 

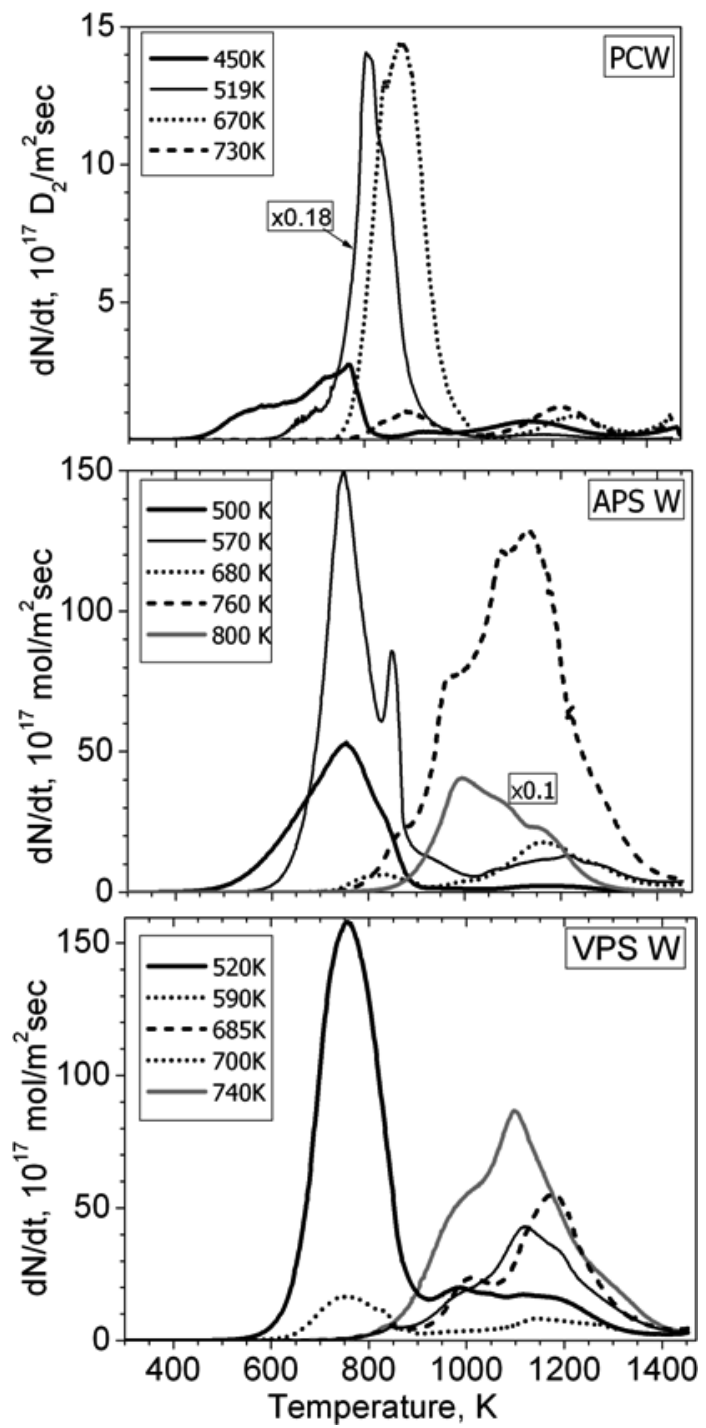

Fig. 3 TDS spectra of $\mathrm{D}_{2}+0.5 \mathrm{HD}$ from PCW, APS W and VPS $\mathrm{W}$ irradiated at various temperatures up to the fluence of $2 \times 10^{25} \mathrm{D} / \mathrm{m}^{2}$

for the fluence of $2 \times 10^{25} \mathrm{D} / \mathrm{m}^{2}$. In the low $T_{\text {irr }}$ region $(<500 \mathrm{~K})$ the retention in PS W is slightly larger, than that in PCW. In the moderate $T_{\text {irr }}$ region (near $600 \mathrm{~K}$ ) the retention in PCW and PSW is similar. In the high $T_{\text {irr }}$ ( $>650 \mathrm{~K}$ ) the behavior of the retention for PS W layers drastically changes compared to PCW. With $T_{\text {irr }}$ increase the retention in PCW quickly decreases while in PS W it increases reaching two orders magnitude difference compared to PCW at $T_{\text {irr }}=750 \mathrm{~K}$. There is no sign of saturation of the retention in APS W up to $T_{\text {irr }}=800 \mathrm{~K}$.

Fluence dependence of the retention in APS W calculated from TDS results in Fig. 4 showed square root behavior with sharp saturation at the fluence of about $4 \times 10^{25} \mathrm{D} / \mathrm{m}^{2}$ (Fig. 6). Note that all points in this dependence had been measured consequently with the same sample. Irradiation with the fluence of $9.8 \times 10^{25} \mathrm{D} / \mathrm{m}^{2}$ showed even smaller retention compared to the fluence of

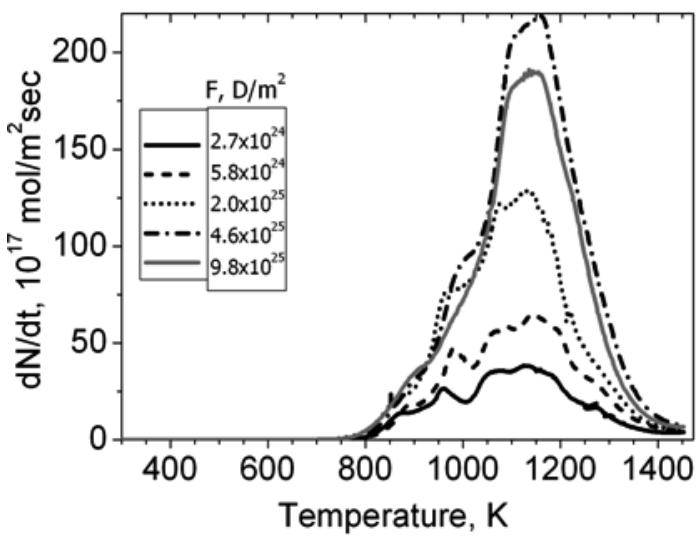

Fig. 4 TDS spectra of $\mathrm{D}_{2}+0.5 \mathrm{HD}$ from APS W irradiated by D plasma up to various fluences. The irradiation temperature is $760 \mathrm{~K}$.

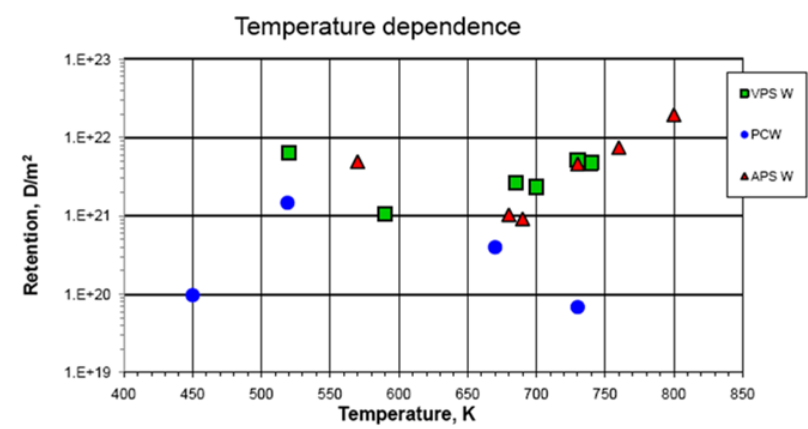

Fig. 5 Temperature dependence of D retention in PCW, APS W and VPS W irradiated by D plasma up to the fluence of $2 \times 10^{25} \mathrm{D} / \mathrm{m}^{2}$.

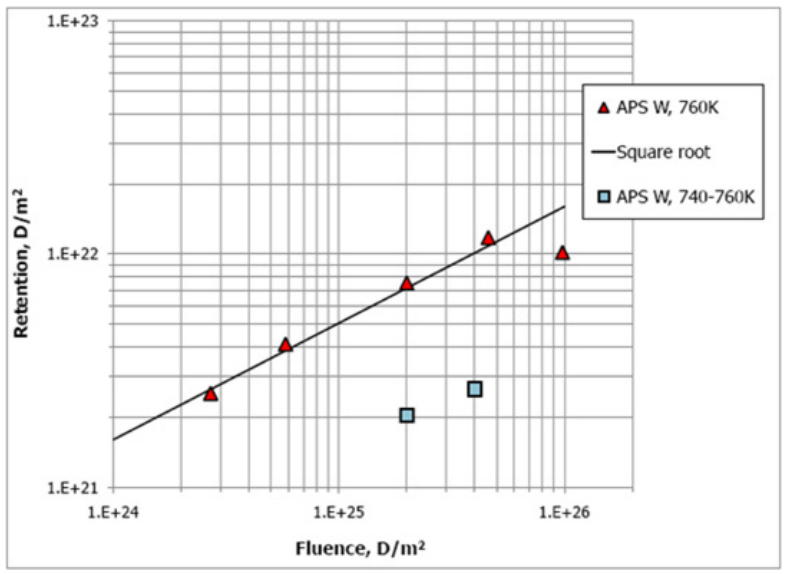

Fig. 6 Fluence dependence of D retention in APS W exposed to $\mathrm{D}$ plasma at $760 \mathrm{~K}$. The repetitive points are shown by squares, the square root behavior is shown by the line.

$4.6 \times 10^{25} \mathrm{D} / \mathrm{m}^{2}$. Moreover, after a set of experiments with this sample at $T_{\text {irr }}=750 \mathrm{~K}$ the repetitive measurements of the retention for the fluences of $2 \times 10^{25} \mathrm{D} / \mathrm{m}^{2}$ and $4 \times 10^{25} \mathrm{D} / \mathrm{m}^{2}$ showed 4 times less values (Fig. 6), 


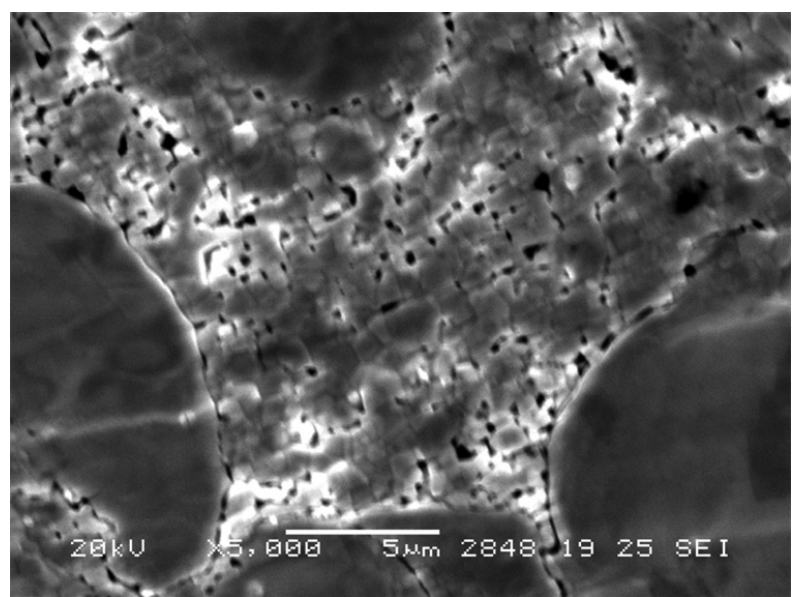

Fig. 7 SEM picture of APS W chemically polished surface.

though the peak positions of the corresponded TDS spectra as well as their shape remained same compared to the earlier measurements performed after irradiation with similar fluences.

An example of scanning electron microscope (SEM) picture of chemically polished surface of APS W is shown in Fig. 7. It is seen from the figure that the material consists of tungsten droplets with the voids between them. Sometimes the droplets contain a lot of small bubbles or voids. The system of voids forms the pores or paths for the migration of deuterium atoms and molecules from the surface inside the bulk. Thus, in order to release to the vacuum from the bulk deuterium can migrate in the form of molecules or atoms along the internal surfaces toward the geometrical surface. Besides, closed pores and voids exist in the material. Deuterium gas accumulated in that space needs to overcome the surface barrier to enter the bulk material and then to release to vacuum or to open pores. Most probably, the internal surfaces of the pores and voids are enriched by the impurities that accumulated on the surface layer of each grain during the deposition process.

Usually, the desorption of hydrogen isotopes implanted in polycrystalline $\mathrm{W}$ from ion beam as well as from plasma has been observed within $300 \mathrm{~K}$ - $1000 \mathrm{~K}$ region [3-6]. It is believed that desorption within $500-600 \mathrm{~K}$ region can be attributed to trapping at low energy defects (dislocations and dislocation loops), the desorption within $600-700 \mathrm{~K}$ [3] to trapping at vacancy clusters and near $900 \mathrm{~K}$ to desorption from chemisorption sites on the surface of voids [4]. The ramp rate in [3] and [4] was larger than that in our experiments, therefore TDS peak positions can differ from those in our results. However, the peak positions can be shifted to higher temperatures with the ramp rate but not to lower temperatures. This means that the higher temperature peak positions in our results compared to the literature observations cannot be explained by different ramp rate. Investigations show that the retention inevitably decreases at high $T_{\text {irr }}$ [7]. This decrease is ex- plained by the larger probability of the release out of the traps at higher $T_{\text {irr }}$ and by the decrease in the point defects concentration due to larger mobility of these defects. Contrary to the polycrystalline tungsten material, high temperature $(>1000 \mathrm{~K}$ ) desorption from plasma sprayed tungsten layers has been reported in several studies [8-10].

In [11] it is suggested that high temperature desorption at high $T_{\text {irr }}$ can be explained by trapping in the substrate material (carbon composite) rather than trapping inside the PS W layers. Therefore, high temperature part of TDS from PS layers in Fig. 3 might be explained by the retention in the substrate. Literature data survey shows that high temperature desorption is indeed typical for the carbon materials [12] but it is not typical for the $\mathrm{F} 82 \mathrm{H}$ steel [13]. In [14] Otsuka et al. have measured tritium depth profiles in plasma exposed APS and VPS W layers deposited on F82H by means of tritium imagine plate technique. It is shown that for $T_{\text {irr }}=573 \mathrm{~K}$ the contribution of the $\mathrm{F} 82 \mathrm{H}$ substrate in the deuterium retention decreases compared to $T_{\text {irr }}=453 \mathrm{~K}$. And for both $T_{\text {irr }}$ this contribution is negligible compared to the deposited layer. Even at the $T_{\text {irr }}=453 \mathrm{~K}$ tritium distributed throughout whole $1 \mathrm{~mm}$ deposited APS and VPS layers. This depth is extremely large as far as deuterium permeation inside $\mathrm{W}$ material at these conditions is concerned. Deep tritium migration was attributed to tritium diffusion (penetration) through open pores and/or migration along grain boundaries followed by adsorption on grain surfaces facing the pores and dissolution of tritium inside the grains.

Thus, one can suggest that PS W layers have additional high energy trapping sites together with those typical for polycrystalline tungsten. Complicate structure of TDS spectra from these layers indicates that there are multiple traps with different detrapping energies. Comparison of high temperature part of TDS spectra from APS and VPS W layers leads to the conclusion that the materials have high energy trapping sites of similar nature. The difference in the relative amplitudes in this TDS region can be explained by different traps concentrations in the material regions involved in the trapping. At low $T_{\text {irr }}$ the trapping mechanisms in PS W layers are mainly similar to those in the polycrystalline $\mathrm{W}$, though small part of $\mathrm{D}$ atoms is trapped in the high energy traps. But with $T_{\text {irr }}$ increase the role of the high energy traps increases substantially in PS W layers. This allows us to suggest that at higher $T_{\text {irr }} \mathrm{D}$ atoms can reach and be trapped in the regions inaccessible at low $T_{\text {irr. }}$.

In [10] it was found that chemical reacted oxygen (W$\mathrm{O}$ bonds) and chemisorbed oxygen (O-O bonds) exist in the surface and interior of VPS tungsten coatings. The amount of chemisorbed oxygen on the coating surface was much more than that of its interior. Increase in the desorption temperature in comparison with tungsten has also been reported for $\mathrm{WO}_{3}$ films [15]. Our EDS measurements showed that the oxygen concentration on APS W surface was reduced about two times within the plasma irradiated 


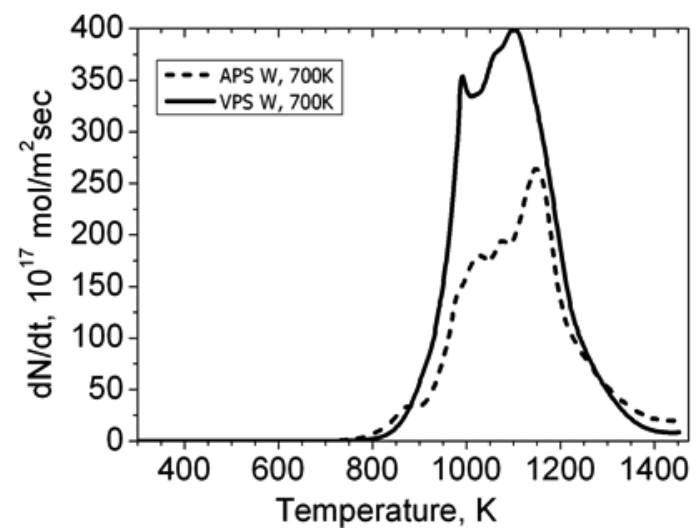

Fig. 8 TDS spectra of chemically polished APS and VPS W, irradiated by $\mathrm{D}$ plasma. The irradiation temperature is $700 \mathrm{~K}$, the fluence is $2 \times 10^{25} \mathrm{D} / \mathrm{m}^{2}$.

area compared to non-irradiated area.

Therefore, following speculations of the trapping mechanisms can be proposed. During plasma irradiation fluxes of ions and neutrals penetrate inside the $\mathrm{W}$ droplets on the top surface. At low $T_{\text {irr }} \mathrm{D}$ atoms cannot migrate to deep regions, therefore the $\mathrm{D}$ is trapped inside the top surface $\mathrm{W}$ droplets and the retention is similar to PCW. However, at higher $T_{\text {irr }} \mathrm{D}$ concentration in lower energy traps corresponded to $800 \mathrm{~K}$ peak in Fig. 3 decreases and $\mathrm{D}$ atoms are able to migrate to deeper regions. Square root behavior of the retention presented in Fig. 6 indicates the diffusion like permeation that is also valid for the defects case. In the deeper regions $\mathrm{D}$ can be trapped inside the closed pores or voids which surfaces are enriched by impurities. The higher the $T_{\text {irr }}$ the larger the number of traps that $\mathrm{D}$ can reach and fill. Existence of higher trapping energy sites in PS W compared to PCW is proved by TDS data. Higher trapping energy sites can explain an increase of the retention in PS W within $650-800 \mathrm{~K} T_{\text {irr }}$ region compared to decrease in PCW presented in Fig. 5. Besides, increase of the retention with $T_{\text {irr }}$ can be also explained by more effective adsorption from gas phase throughout whole PS layer thickness as it was proposed in [14]. However, the contribution of this process in the total desorption is difficult to estimate as the dissociation and recombination coefficients and the D pressure inside the pores are unknown.

One can suggest that the mechanism of trapping is simple and involves trapping only inside the surface layer directly contacted with plasma ions. TDS spectra of deuterium from APS and VPS W, which surfaces were vanished by chemical polishing and irradiated at $T_{\mathrm{irr}}=750 \mathrm{~K}$, showed similar structure compare to as received samples (Fig. 8). The spectra still contain large high temperature part with numerous peaks and the retention became even higher (Fig. 8). This proves that the retention is determined not only by the geometric surface but also by the internal structure (including surfaces of cracks and pores) of plasma sprayed layers.
After deposition the PS W layers hadn't been annealed whereas PCW sample had been annealed in vacuum at $1173 \mathrm{~K}$ during 1 hour. Therefore, each W drop of PS layers is likely to contain much larger amount of defects compared to the polycrystalline sample. This can lead to a speculation about delayed D release from deeper regions of PS W and consequently high desorption temperatures. This means that the detrapping energies could be not as high as it was suggested. In order to clarify this possibility the "delay" experiment has been performed. In this experiment the linear heating of APS W irradiated by plasma at $T_{\text {irr }}=750 \mathrm{~K}$ up to the fluence of $2 \times 10^{25} \mathrm{D} / \mathrm{m}^{2}$ was stopped at the temperature of $800 \mathrm{~K}$ and kept at this level during 2 hours. D atoms were partially desorbed from the sample, but not completely. After the delay the linear heating was continued and substantial part of deuterium was desorbed in the high temperature region (1000 - 1400 K). This means that $\mathrm{D}$ atoms are indeed trapped by strong traps during plasma irradiation at high $T_{\text {irr }}$. In order to remove deuterium from the material it has to be heated up to high temperatures, and this could be critical for using the material as plasma facing in the fusion reactor.

The reason for the reduction of the deuterium retention in the repetitive experiments (Fig. 6) is unclear yet. Two possibilities can be suggested. The first one is the defects annealing during TDS runs. Indeed, gradual defects annealing during multiple TDS runs might take place as it was observed in [5]. Our experiments with multiple irradiation $\left(T_{\mathrm{irr}} \approx 730 \mathrm{~K}\right)$ and thermal desorption of one APS W showed increased deuterium retention only for the first irradiation. Two subsequent irradiations showed almost constant retention. It was not changed even after subsequent annealing at $1173 \mathrm{~K}$ during 1 hour. Therefore, defects annealing in TDS runs cannot explain the mentioned reduction of the retention. Another possibility is a decrease of high traps concentration due to plasma exposure. This could be explained by partial cleaning of the contaminated internal surfaces during long time deuterium irradiation and thermal desorption. Decrease of oxygen concentration on the surface of APS W might be attributed to this effect.

Comparison with existed data on the retention in PS W layers shows substantial scattering. In [11] constant retention in VPS W on graphite was measured in the irradiation temperature region of $350-800 \mathrm{~K}$. The retention was at the level of $10^{21} \mathrm{D} / \mathrm{m}^{2}$ and in the low temperature region the results are similar to the present work: the retention reduces with the irradiation temperature. On the other hand, further increase of the irradiation temperature didn't show reduction or increase of the retention. In [9] the deuterium retention in two types of plasma sprayed coatings has been investigated, namely inert gas plasma sprayed (IPS) and VPS. It is shown that the structure of deuterium TDS spectra of these coatings exposed at $T_{\mathrm{irr}}=590 \mathrm{~K}$ and $T_{\text {irr }}=715 \mathrm{~K}$ differs substantially. In [16] the retention in VPS W exposed to high flux plasma showed an increase at high irradiation temperatures while it was reduced for 
polycrystalline $\mathrm{W}$ samples. But the retention level was much less compared to our results. Thus, the importance of impurities presence on the internal surfaces and therefore the structure of plasma sprayed layer itself could explain the above results and data scattering. It seems, the manufacturing/deposition process, the $\mathrm{W}$ drops contamination level, the sprayed layer quality (e.g. the pores density) should have strong influence on the retention properties.

At high plasma irradiation temperatures plasma sprayed layers can store huge amount of hydrogen isotopes. In order to remove it from the material high heating temperatures are necessary. The internal structure of the layers plays crucial role in the hydrogen isotopes accumulation. Therefore, it is quite important to find optimal regimes of deposition when the number and the size of pores and cracks between $\mathrm{W}$ drops and the contamination level of the drops is minimal.

\section{Conclusions}

Deuterium trapping at low energy plasma irradiation in plasma sprayed layers was investigated by means of thermal desorption spectroscopy. It was observed that the retention in the plasma sprayed layers strongly increases at high irradiation temperatures contrary to the polycrystalline tungsten. It is the high temperature part $(>1000 \mathrm{~K})$ of TDS that gives a large contribution in the total retention after high temperature irradiations. Such high desorption temperatures are not typical for the polycrystalline tungsten and can be explained by different internal structure of the materials. It was suggested that the retention in the plasma sprayed layers at high irradiation temperatures is determined by the trapping at internal surfaces enriched with impurities. Extremely large deuterium retention in the plasma sprayed layers is caused by huge surface area of numerous pores and cracks existed in between the $\mathrm{W}$ drops constituted the layers. It was found that the number of trapping sites is reduced with long time irradiation at high temperatures though the possible mechanism is not clear yet.

This high storage ability of hydrogen and high detrapping energies could be negative for using this material as plasma facing in the fusion reactor especially at elevated divertor working temperatures. In order to improve their characteristics it is necessary to reduce the number of voids and cracks and contamination level in the plasma sprayed layers by optimization of the deposition procedure.

[1] J. Matějíček, P. Chráska and J. Linke, Thermal Spray Coatings for Fusion, Applications - Review, Journal of Thermal Spray Technology 16(1), 64 (2007).

[2] S.K. Sharma, H. Zushi, N. Yoshida et al., Fusion Eng. Des. 87, 77 (2012).

[3] M. Poon, A.A. Haasz and J.W. Davis, J. Nucl. Mater. 374, 390 (2008).

[4] O.V. Ogorodnikova, J. Roth and M. Mayer, J. Nucl. Mater. 313-316, 469 (2003).

[5] A. Rusinov, Yu. Gasparyan, N. Trifonov et al., J. Nucl. Mater. 415, S645 (2011).

[6] J.P. Sharpe, R.D. Kolasinski, M. Shimada et al., J. Nucl. Mater. 390-391, 709 (2009).

[7] V. Kh. Alimov and J. Roth, Physica Scripta T128, 6 (2007).

[8] O.V. Ogorodnikova, T. Schwarz-Selinger, K. Sugiyama et al., Physica Scripta T138, 014053 (2009).

[9] V.Kh. Alimov, H. Nakamura, B. Tyburska-Püschel et al., J. Nucl. Mater. 414, 479 (2011).

[10] Y. Niu, S. Suzuki, X. Zheng et al., J. Nucl. Mater. 417, 551 (2011).

[11] V.Kh. Alimov et al., J. Nucl. Mater. 415, S628 (2011).

[12] T. Suda, H. Miyauchi, A. Yoshikawa et al., Fusion Eng. Des. 82, 1762 (2007).

[13] T. Ito, Y. Yamauchi, T. Hino et al., J. Nucl. Mater. 417, 1147 (2011).

[14] T. Otsuka, T. Tanabe and K. Tokunaga, Physica Scripta T145, 014035 (2011).

[15] N. Matsunami, N. Ohno and M. Tokitani, J. Nucl. Mater. 390-391, 693 (2009).

[16] G.-N. Luo et al., Nucl. Instrum. Methods in Phy. Res. B 267, 3041 (2009). 